\title{
Pulse Jitter, Delay Spread and Doppler Shift in Mode-Stirred Reverberation
}

\author{
Luk R. Arnaut
}

\begin{abstract}
A time-domain characterization and stochastic model are developed for analyzing the evolution of the early randomization of fields inside mode-stirred reverberation chambers. The chamber is excited by a pulse amplitude modulated RF signal and stirred mechanically by a rotational stirrer. Phase noise (jitter) in the arrival times of a received pulse train is found to contain a systematic contribution that depends on stir speed and stir sense, as well as a random contribution governed by the rate of field depolarization. For the systematic component, a model of the phase delay for a uniformly orbiting scatterer inside a cavity is derived. For the random component, a nonhomogeneous Poisson model for arrivals of wave fronts in a nonstationary stir process exhibits good agreement with the empirical distribution of the measured jitter. At increasing stir velocities, the rate of field mixing increases less rapidly. The excess delay approaches asymptotically an Erlang distribution, in the limit of a homogeneous Poisson process for uniformly distributed late arrival times.
\end{abstract}

Keywords: electromagnetic pulse, jitter, mode-stirred reverberation, phase noise

\section{INTRODUCTION}

Dynamic multipath propagation is a paradigm of both fundamental and practical importance in wireless transmission of signals and power, e.g., for intervehicular communications in urban scenarios. For signals in such continuously timevarying electromagnetic environments (EMEs), fields can be emulated using a modulated source inside a reverberation chamber. In certain cases, the time scale of the excitation signal and the response times of the instrumentation may have comparable orders of magnitude. The latter include the characteristic time constants of the unperturbed cavity (modal decay or relaxation times), the inverse rate of stirring (field coherence time), the acquisition times of the field sensors, filters, detectors, analyzers, etc. In a time-varying resonant EME, such 'interference' of time scales can give rise to a complex time-frequency behavior of the observed field and signal distortion.

In a static cavity, the probability distribution of the spectral spacing of resonance frequencies enables a classification of wave chaos based on random matrix theory [1]. In a modetuned reverberation chamber (MTRC), i.e., whose boundary changes at discrete times only, its resonances remain sharply defined because the field varies quasi-statically. The evolution of these resonances through successive cavity states can be tracked by spectrograms, showing the trajectories of the resonance frequencies (spectral localization) [2, Fig. 13], [3, Fig. 2], [4] or their spacings, widths, velocities [5, Fig. 4], or other spectral characteristics as a function of the discrete perturbation (stir) parameter. Statistical characterization of these changes then enables further classification.
In a dynamic mode-stirred reverberation chamber (MSRC), the continuous morphing of the cavity boundary produces a nonstationary perturbation of the interior field. In this case, the spectrogram - with stir speed and acceleration as parameters - exhibits diffusion (blurring) of the previously sharp timefrequency transitions and trajectories for the resonance dynamics and the short-term spectrum. Consequently, the spectrum must now be tracked in its entirety, for a continuum of frequencies. This makes a classical spectral stochastic characterization problematic. In essence, the issue is caused by the fact that wave resonance is an inherently steady-state concept, based on standing waves that are formed by counterpropagating waves travelling along closed paths in a strictly static (time-invariant) configuration.

When the mechanical stir rate is nonzero, additional spectral perturbations arise that depend on this rate, viz., Doppler shifts and spectral broadening with their time variations. These are of course more pronounced in acoustic MSRCs [6]-[8], where the wave velocity is orders of magnitude lower than the the speed of light, c, in electromagnetic (EM) reverberation. In the latter case, the shifts and spreads are merely of the order of $100 \mathrm{~Hz}$ typically, which is much smaller than typical modal and average effective Q-bandwidths. This makes their direct observation more difficult [9]. Nevertheless, even such small shifts may produce significant phase noise impairment in wireless communications. For example, multi-carrier OFDM exhibits densely spaced subcarriers, for which Doppler shifts of this magnitude can become comparable to the width and spacing for the subcarrier modulation. More generally, continuous ultra-wideband (UWB) spectra may exhibit different shifts for different excitation frequencies, causing distortion of the signal spectrum. Particularly in multi-mode MSRCs, the simultaneously excited modes may all exhibit widely different relative Doppler shifts. Therefore, the study of even relatively low stir speeds and accelerations in EM MSRCs is of interest.

Specifically relating to phase or frequency modulation, a major motivation for the present work is the characterization of the sensitivity of early-time phase noise to dynamic multipath propagation, which is important in real-time demodulation and decoding. This issue is encountered in techniques that rely on phase discrimination of received waveforms for PPM, PWM, PSK, APSK, OFDM, etc., particularly for coding using a large set of symbols for which detection thresholds are closely spaced and where noise margins are tight. More accurate early characterization of nonstationary multipath propagation can lead to lower symbol error rates and higher channel capacity. For example, in OFDM the prefix guard interval occupies a fixed minimum length or proportion of the active symbol duration for combating reflection echoes that would 
otherwise generate intersymbol and intercarrier interference. Consequently, jitter at the start of the symbol waveform also affects the detected phase of the active interval. Hence, precise characterization of jitter results in low phase errors, bit error rates and their uncertainties. This increased precision may also help to shorten the guard interval, to lengthen the active and overall symbol intervals, and to increase the channel capacity.

In this paper, it is shown that a probabilistic characterization of the delay spread in the arrival times of modulated signals in MSRCs enables an amplitude-free evaluation and characterization of the field randomization process. It avoids difficulties of separating the effects of local averaging and Doppler shifting on the field envelope. Both systematic and random jitter [10] are shown to provide information on the stir parameter values and on the quality of stirring. In addition, it is shown how pulse modulated excitation reveals the interplay between the dynamics of the physical environment and those created by intrinsic EM transients associated with pulse edges. Our analysis relies on a full probabilistic characterization of the received signal, as afforded by the high sampling rates that are achievable by state-of-the-art network and spectrum analyzers and oscilloscopes, which enable real-time I/Q sampling of rapid fluctuations.

This paper extends previous work on early-time characterization of pulses in quasi-static chambers [11], harmonic or pulse modulated excitation of nonstationary chambers [12], [13, Sec. 10.2.3], and a study of more general wave diffusion in nonstationary EMEs [14], [15]. Short-, medium- and longterm characterization of power decay and steady state in static and quasi-static reverberant rooms has typically focused on amplitude-based characterization and has a long history, e.g., [16]-[19]. Summary accounts of selected results from this paper were presented in [12] and [20].

\section{Time Delay and Doppler Shift fOR SCATterers IN UNIFORM MOTION INSIDE CAVITY RESONATORS}

In an overmoded dynamic 3-D stirred cavity, the tracking of multiple cavity modes or paths of propagation and the associated temporal evolution of the delay spread or Doppler shifts is difficult. Considerable insight can be gained by first studying harmonic excitations of a dynamic 1-D resonator instead, which displays many of the salient features.

Consider a 1-D perfectly electrically conducting (PEC) parallel-plate (Fabry-Pérot) resonator with wall separation distance $d$ that may vary with time $t$ (Fig. 1a). A harmonic plane-wave source is located at the left wall $(x=0)$ and a receptor (dot sensor or scatterer) is placed at $x=r$ $(0<r<d)$. At least four scenarios of wall dynamics are feasible: (i) one fixed and one moving wall (translatory and/or oscillatory or randomly), (ii) both walls moving independently (asynchronously), (iii) both walls moving periodically, at the same rate but out of phase (constant mechanical phase difference), and (iv) both walls moving periodically and in phase (synchronously). The first three cases imply a time-varying width $d(t)$ and time-varying modal resonance frequencies, in the quasi-static approximation. The fourth scenario maintains a constant $d$ but yields a time-varying phase of the sensed field. An equivalent case is that of static boundaries with a moving receptor, which is analyzed first in Sec. II-A. In Sec. II-B, this is then applied to realistic mode stirring. In Sec. III, a modulated source and a different, more practical definition of delay are adopted in an experimental study. Deterministic motion of the walls is assumed but can be extended to random motion, as e.g. in the electric analog of second-order Fermi acceleration.

Fig. 1. (a) Rectilinear and (b) circular motion of a receptor inside a 1-D cavity.

\section{A. Rectilinear Receptor Motion}

1) Static Receptor: For a harmonic EM plane-wave source of frequency $\omega \equiv k$ c polarized parallel to the PEC boundaries, the unit amplitude field received by a receptor at $r(t)$ is

$$
\begin{aligned}
E(r, t, \omega) & =\sin [\omega t-k r(t)]-\sin [\omega t-k(2 d-r(t))] \\
& \triangleq \sin \Psi_{+}-\sin \Psi_{-} .
\end{aligned}
$$

For a receptor that is at rest relative to the cavity $\left(r(t)=r_{0}\right)$, the relative (excess) time delay between the local forward and reflected backward wave fields is independent of time, i.e.,

$$
T_{d, 0}=T_{+}-T_{-} \triangleq \frac{\Psi_{+}-\Psi_{-}}{\omega}=\frac{2\left(d-r_{0}\right)}{\mathrm{c}}
$$

where the subscript 0 in $T_{d, 0}$ indicates zero relative motion. The coherence bandwidth, across which the amplitude changes from a maximum to a minimum, is then also constant, i.e.,

$$
B_{c, 0} \triangleq \frac{1}{T_{d, 0}}=\frac{\mathrm{c}}{2\left(d-r_{0}\right)} .
$$

2) Receptor in Uniform Relative Motion (Constant Velocity): For a receptor at $r(t)=r_{0}+v t$ with time-independent $v$, the Doppler frequency shift (in units $\mathrm{Hz}$ ) is

$$
\begin{aligned}
D_{v} & \equiv-\left(D_{+}-D_{-}\right) \triangleq-\frac{\omega}{2 \pi}\left(\dot{T}_{+}-\dot{T}_{-}\right) \\
& =\frac{\dot{\Psi}_{-}-\dot{\Psi}_{+}}{2 \pi}=2 \beta f \equiv \frac{2 v}{\lambda}
\end{aligned}
$$

where $\dot{\Psi}_{ \pm} \equiv \mathrm{d} \Psi_{ \pm} / \mathrm{d} t=\omega \mp k v$ and $\beta \triangleq v / \mathrm{c}(>0$ or $<0)$. In (4), the sign was chosen such that a receptor moving towards the source in the direction of negative coordinates experiences 
an increase of frequency. The associated coherence time is then

$$
T_{c, v}=\frac{1}{2 D_{v}}=\frac{1}{4 \beta f} .
$$

From (2), the effect of a uniform velocity on the phase delay and delay spread is a linear dependence on time, i.e.,

$$
\begin{aligned}
T_{d, v}(t) & =\frac{\Psi_{+}(t)-\Psi_{-}(t)}{\omega}=\frac{2[d-r(t)]}{\mathrm{c}} \\
& =T_{d, 0}-2 \beta t \equiv T_{d, 0}-\frac{t}{2 f T_{c, v}},
\end{aligned}
$$

which shows an explicit relationship between coherence time and delay spread. Consequently, compared to $T_{d, 0}$, timecontinuous uniform mode stirring causes the delay spread to increase $(\beta<0)$ or decrease $(\beta>0)$ linearly with time, at a rate inversely proportional to the coherence time. Specifically, a positive speed increment $\Delta v=\left(r-r_{0}\right) / \Delta t \equiv \mathrm{c} \Delta \beta>0$ shortens the delay spread by $\Delta T_{d, v}=-2(\Delta \beta) t$.

We shall further adopt the quasi-static approximation for nonrelativistic motion $(|\beta| \ll 1)$. To first order, the coherence bandwidth associated with (7) then varies linearly with time as

$$
B_{c, v}(t)=\frac{1}{T_{d, v}(t)} \simeq \frac{1}{T_{d, 0}}\left(1+\frac{2 \beta}{T_{d, 0}} t\right),
$$

where the approximation holds for $t \ll T_{d, 0} / \beta$. With (7), this also relates $B_{c, v}$ to $T_{c, v}$. Thus, a moving sensor or scatterer with increasing $\Delta v(>0)$ causes the relative coherence bandwidth to increase linearly with time by $\Delta B_{c, v}(t) / B_{c, 0}=$ $2 \beta t / T_{d, 0}=-\Delta T_{d, v}(t) / T_{d, 0}$. If the speed is reduced $(\Delta v<$ 0 ), the changes in the delay and bandwidth occur in the opposite sense, i.e., delay dilation and bandwidth reduction.

On eliminating the dependencies on $d-r_{0}$ and $\beta$, a relationship between coherence bandwidth and Doppler shift follows: eq. (8) together with a Taylor series expansion of $1 /\left[1-v t /\left(d-r_{0}\right)\right]$ in (7) yields

$$
B_{c, v}(t) \simeq B_{c, 0}+\left(\frac{2 B_{c, 0}^{2}}{f} D_{v}\right) t .
$$

3) Uniformly Accelerating Receptor: Now assume a pair of synchronously moving and accelerating walls, or an accelerating sensor or scatterer between static walls, e.g., instantaneously during an oscillatory motion of the receptor. With $r(t)=r_{0}+v_{0} t+a t^{2} / 2$, the quadratic time dependence now produces new terms: substituting $r(t)$ into (1) yields the received field as

$$
\begin{aligned}
E(t, \omega)= & \sin \left[-\frac{k a}{2} t^{2}+k \mathrm{c}\left(1-\beta_{0}\right) t-k r_{0}\right] \\
& -\sin \left[\frac{k a}{2} t^{2}+k \mathrm{c}\left(1+\beta_{0}\right) t-k\left(2 d-r_{0}\right)\right],
\end{aligned}
$$

where $\beta_{0} \triangleq v_{0} / \mathrm{c}$ is the relative initial velocity at $t=0$. An additional coefficient now arises that is governed by the linear rate of frequency change (chirp difference, i.e., uniform acceleration of phase) and can be defined as

$$
A_{a} \equiv-\left(A_{+}-A_{-}\right)=\frac{2 a}{\lambda} \triangleq 2 \gamma f
$$

where $A_{ \pm} \triangleq \ddot{\Psi}_{ \pm}(t) /(2 \pi)=\mp k a /(2 \pi)=\mp a / \lambda$ based on (10), with $\gamma \triangleq a / c(>0$ or $<0)$. Thus, accelerated motion increases the absolute rate of frequency shifting $2|\gamma| \equiv$ $\left|\mathrm{d} A_{a} / \mathrm{d} f\right|$. Since $v(t)=v_{0}+a t$, the initial Doppler shift and delay spread remain formally the same as (4) and (6) for uniform motion, i.e., $D_{a}(0)=D_{v_{0}}=2 \beta_{0} f$ and $T_{d, a}(0)=$ $T_{d, v_{0}}(0)=T_{d, 0}$, respectively, while their instantaneous values $D_{a}(t)$ and $T_{d, a}(t)$ follow by replacing $\beta$ with $\beta(t) \equiv v(t) / c$. Thus, at later times, the delay spread and coherence bandwidth now exhibit an additional quadratic term, whereas the Doppler shift acquires a linear time dependence, i.e.,

$$
\begin{aligned}
T_{d, a}(t) & =\frac{\Psi_{+}(t)-\Psi_{-}(t)}{\omega}=\frac{2\left[d-\left(r_{0}+v_{0} t\right)\right]-a t^{2}}{\mathrm{c}} \\
& =T_{d, 0}-2 \beta_{0} t-\gamma t^{2} \\
B_{c, a}(t) & =\frac{1}{T_{d, 0}-2 \beta_{0} t-\gamma t^{2}} \\
D_{a}(t) & =-f \dot{T}_{d, a}(t)=2 f\left(\beta_{0}+\gamma t\right) .
\end{aligned}
$$

Similar to (5) - and in analogy with the Fourier pair formed by the delay spread and coherence bandwidth in (3) - a timedomain quantity that is paired with the spectral coefficient (11) follows. From (14), this can be interpreted as a linear chirp dilation (or compression, if negative) with coefficient

$$
C_{a} \triangleq \frac{1}{A_{a}}=\frac{1}{2 \gamma f}
$$

on account of $(k a) t^{2}=(a t / \mathrm{c}) \psi$, where $\psi \triangleq \omega t$ is the reference phase at the location of the source $(r=0)$.

For nonuniform (higher-order) accelerated motion in $r(t)$ and $d(t)$, including jerk, jounce, etc., the expressions for the delays, coherencies, and spreads in time or frequency follow by generalization and substitution. These are particularly relevant during stepping transients of the mode tuner.

\section{B. Stirrer Motion}

Typical mechanical stirring is relatively slow compared to the time variation of the unstirred field and c. Thus, it generates a quasi-static EM process, The instantaneous rest frame hypothesis [21] can therefore be assumed, with which velocity and acceleration are linearized near the static equilibrium at $t=0$.

1) (Quasi-)Uniform Rectilinear Stirring: Linear (1-D) uniform stir motion can be realized by a controlled translatory stirrer (e.g., [22]). If the motion is periodic, then approximate uniformity of stir speed is attained sufficiently far from the extremities (turning points) of the stirrer. The speed is then nearly constant and acceleration is low $\left(|\gamma| t \ll\left|\beta_{0}\right|\right)$. For 1 D simple harmonic stir motion (e.g., pendulum stirring), it is also approximately valid sufficiently close to its equilibrium rest state, at a distance $r_{0}$.

Near an equilibrium of the mechanical phase $\theta(t)=\Omega t$, i.e., for $(\Omega t) \bmod \pi \ll 1$ where $r(t)=r_{0}+s \sin \theta(t) \simeq r_{0} \pm s \Omega t$ and $v(t)=s \Omega \cos \theta(t) \simeq \pm s \Omega$ hold, it follows from (7) that

$$
T_{d}=T_{d, v}(\Omega t) \simeq T_{d, 0} \mp \frac{2 s}{\mathrm{c}} \Omega t
$$

where $T_{d, 0}=2\left(d-r_{0}\right) / \mathrm{c}$, with $r_{0}=s=d / 2$ in the case of wall-to-wall stirring, and where upper and lower signs 
correspond to motion toward or away from the right wall, respectively. Thus, for 1-D uniform or harmonic stirring near equilibrium, the delay spread varies linearly away from $T_{d, 0}$ at a rate governed by the rectilinear velocity, $s \Omega$.

2) Rotational or Nonuniform Rectilinear Stirring: Conventional mechanical mode stirrers are of rotating type. All blades of a rigid stirrer are co-rotating at the same angular velocity $\Omega(t)$, thus forming a "swarm" of elementary scattering facets with individual cross-sections $\delta A \cos \left(\theta+\theta_{0}\right) / \lambda^{2}$ presented to the incident local field. As each facet rotates on a specific radius $s$, its tangential velocity $v=s \Omega$ and centripetal acceleration $a=s \Omega^{2}-$ and, hence, the effect of $v$ and $a$ on the instantaneous $T_{d}, B_{c}$ and $D$ - increase with $s$. Because of the large radial size of a typical stirrer, the multitude of inclinations of the facets relative to their ambient incident field, and the quasi-random inhomogeneity of this field, there exist large variations among the individual contributions of all facets to the overall $T_{d}, B_{c}$ and $D$. Moreover, when the facets move through a quasi-random multi-modal field, their contributions appear as random, even for facets on equal $s$ and uniform rotation. This randomness can be captured by randomizing the radius as $S$, with PDF $f_{S}(s)$. To first order, $s$ can then be replaced by its mean value

$$
s \rightarrow\langle S\rangle \triangleq \int_{s_{\min }}^{s_{\max }} s f_{S}(s) \mathrm{d} s,
$$

but bearing in mind that the functional form of $f_{S}(s)$ may significantly affect the global averages and PDFs of $T_{d}$ and $D$.

Returning to a single facet with 2-D circular uniform rotation on a radius $s$, upon projecting its orbit onto a direction $o x$ in its plane (Fig. 1b), a 1-D harmonic oscillator along $o x$ with linear accelerated motion is obtained. The previous expressions for $r(t), v(t)$, and their first-order approximations for $\theta(t) \simeq m \pi$ remain applicable as $r_{x}(t)$ and $v_{x}(t)$, in conjunction with $a_{x}(t)=-s \Omega^{2} \sin \theta(t) \simeq \mp s \Omega^{3} t$. Conversely, near the walls, $\theta(t) \simeq 2 m \pi \mp \pi / 2, r_{x} \simeq r_{0} \mp s$, $v_{x}(t) \simeq \pm s \Omega^{2} t$, and $a_{x} \simeq \pm s \Omega^{2}$. Consequently, the values and local time dependences of $T_{d}$ and $D$ depend on the particular location and orientation angle of the stirrer, i.e., on the instance of observation during its rotation. For constant $\Omega$ but arbitrary $t$, the delay spread follows from (6) as

$$
T_{d, x}(\Omega t)=T_{d, x, 0}-\frac{2 s}{\mathrm{c}} \sin \Omega t .
$$

The rate of delay spread $\dot{T}_{d, x}$ and Doppler shift $D_{x}=-f \dot{T}_{d, x}$ follow as

$$
\dot{T}_{d, x}=-2 \beta(\Omega) \cos \Omega t, \quad D_{x}=\frac{2 s \Omega}{\lambda} \cos \Omega t .
$$

Since $T_{d, x}$ and $\dot{T}_{d, x}$ are odd functions of $\Omega$, these enable a purely EM based discrimination between clockwise $(\mathrm{CW})$ and counter-clockwise $(\mathrm{CCW})$ stirring $(\Omega>0$ or $<0)$. Fig. 2 shows $\dot{T}_{d, x}(\Omega)$ for $s=1 \mathrm{~m}$ and $t=1 \mathrm{~s}$.

For a 2-D or 3-D cavity, a similar projection onto oy in the plane of circular ${ }^{1}$ rotation yields $T_{d, y}=T_{d, y, 0}+(2 \mathrm{~s} / \mathrm{c}) \cos \Omega t$ and $D_{y}=(2 v / \lambda) \sin \Omega t$. These can be formally combined

\footnotetext{
${ }^{1}$ An extension to elliptic stirring follows by replacing $s$ with $s_{x}$ and $s_{y}$.
}

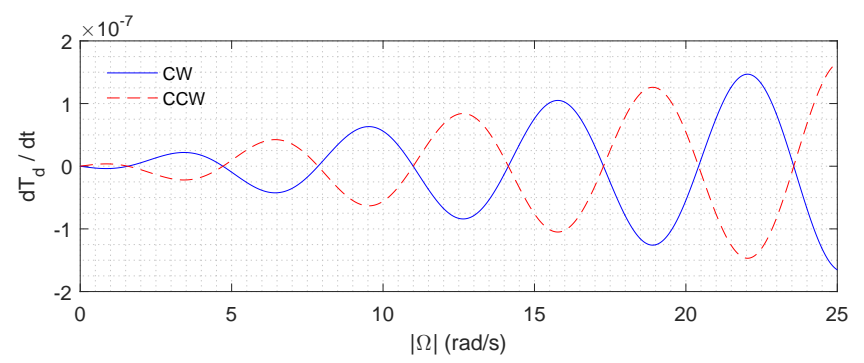

Fig. 2. Rate of time delay spread for scatterer in projected uniform rotational motion as a function of $|\Omega|$ for $s=1 \mathrm{~m}$ and $t=1 \mathrm{~s}$. Blue solid and red dashed curves correspond to clockwise $(\Omega<0)$ and counterclockwise $(\Omega>0)$ rotations, respectively.

with $T_{d, x}$ and $D_{x}$ to complex-valued quantities $T_{d}=T_{d, x}+$ $\mathrm{j} T_{d, y}$ and $D=D_{x}+\mathrm{j} D_{y}$ as

$$
T_{d}=T_{d, 0}+\mathrm{j} \frac{2 s}{\mathrm{c}} \exp (\mathrm{j} \Omega t), \quad D=\frac{2 s \Omega}{\lambda} \exp (\mathrm{j} \Omega t)
$$

where $T_{d, 0} \equiv T_{d, x, 0}+\mathrm{j} T_{d, y, 0}=2\left[\left(d_{x}-r_{0, x}\right)+\mathrm{j}\left(d_{y}-r_{0, y}\right)\right] / \mathrm{c}$. If $T_{d, x}$ and $T_{d, y}$ add incoherently, through contributions to $T_{d}$ that are statistically independent and weighted equally, then the RMS values $T_{d}^{(\mathrm{i})}=\sqrt{T_{d, x}^{2}+T_{d, y}^{2}}$ and $D^{(\mathrm{i})}=-f \dot{T}_{d}^{(\mathrm{i})}$ are

$$
\begin{aligned}
& T_{d}^{(\mathrm{i})}=T_{d, 0}^{(\mathrm{i})} \sqrt{1-\frac{4 s}{\mathrm{c} T_{d, 0}^{(\mathrm{i})}}\left(\frac{T_{d, x, 0}}{T_{d, 0}^{(\mathrm{i})}} \sin \Omega t-\frac{T_{d, y, 0}}{T_{d, 0}^{(\mathrm{i})}} \cos \Omega t\right)}, \\
& D^{(\mathrm{i})}=\frac{\frac{2 s \Omega}{\lambda}\left(\frac{T_{d, x, 0}}{T_{d, 0}^{(i)}} \cos \Omega t+\frac{T_{d, y, 0}}{T_{d, 0}^{(\mathrm{i})}} \sin \Omega t\right)}{\sqrt{1-\frac{4 s}{\mathrm{c} T_{d, 0}^{(\mathrm{i})}}\left(\frac{T_{d, x, 0}}{T_{d, 0}^{(i)}} \sin \Omega t-\frac{T_{d, y, 0}}{T_{d, 0}^{(\mathrm{i})}} \cos \Omega t\right)}}
\end{aligned}
$$

where $T_{d, 0}^{(\mathrm{i})} \triangleq \sqrt{\left|T_{d, 0}\right|^{2}+(2 s / \mathrm{c})^{2}}$. Alternatively, for coherent superposition, $T_{d}^{(\mathrm{c})}=T_{d, x}+T_{d, y}$ and $D^{(\mathrm{c})}=-f \dot{T}_{d}^{(\mathrm{c})}$ are

$$
\begin{aligned}
T_{d}^{(\mathrm{c})} & =T_{d, 0}^{(\mathrm{c})}\left[1-\frac{2 s}{\mathrm{c} T_{d, 0}^{(\mathrm{c})}}(\sin \Omega t-\cos \Omega t)\right], \\
D^{(\mathrm{c})} & =\frac{2 s \Omega}{\lambda}(\cos \Omega t+\sin \Omega t)
\end{aligned}
$$

where $T_{d, 0}^{(\mathrm{c})} \triangleq T_{d, x, 0}+T_{d, y, 0}$. Unlike in (19) and (20), the coefficients of $2 s \Omega / \lambda$ in (22) and (24) exhibit non-unit amplitudes. This indicates that different maximum Doppler shifts may be expected in stirred 3-D cavities [23, Figs. 3 and 4] compared to the well-known free-space value $D=2 s \Omega / \lambda$ in continuous-wave radar, from (16). Finally, replacing $s$ with $S$ and multiplying with an $s$ - and frequency-dependent scattering cross-section of the facets, followed by an integration as in (17), yields $\left\langle T_{d}(\Omega t)\right\rangle$ and $\langle D(f, \Omega, t)\rangle$ as average (effective) values of the random $T_{d}(\Omega t)$ and $D(f, \Omega, t)$ [24].

Since higher-order cavity modes exhibit spatial variation along both $o x$ and $o y$, rotational stirring affects the overall delay spread through interaction of the moving scatterer with the standing wave functions for both spatial components of these modes. In such interaction, the spatial harmonicity of 
each mode ( $k x, k y$ dependence) "interferes" with the temporal harmonicity ( $\Omega t$ dependence) in the 2-D rotational stir process.

\section{Measurements}

Because of the time-frequency dichotomy, it is preferential to perform measurements in either the time or frequency domain, as opposed to a hybrid representation, e.g. shortterm spectra. Since $D=-f \dot{T}_{d}$, Doppler characteristics follow immediately once $\dot{T}_{d}$ is determined. Time delay and delay spread can be experimentally determined using a digitally modulated RF source signal, for which the start of the modulated pulse provides a time marker for the reception of multipath components.

The $6.55 \times 5.85 \times 3.5 \mathrm{~m}^{3}$ chamber used for the experiments is the same as in [11], but is here operated as a MSRC, i.e., with continuous rotation of its paddle wheel (mode stirrer) at constant rotation speed $\Omega$, further expressed in revolutions per second (rps). The dynamic-to-static surface area and swept volume ratios are $\xi_{S}=9.9 \%$ and $\xi_{V}=4.1 \%$, respectively, as measures of potential stirring capability of the chamber. In our set-up, a signal generator (Hewlett Packard E4433B ESG-D) produces a $2.5 \mathrm{GHz}$ carrier. Its amplitude is then pulse modulated by a square wave generator (Berkeley Nucleonics 6040 (201E); rise time 150 ps) with pulse repetition frequency $1 / T_{0}=10 \mathrm{kHz}$, pulse period $T_{0}=100 \mu \mathrm{s}$, and duty cycle $40 \%$. Two identical transmitting and receiving dual-ridge horn antennas (EMCO 3115) are separated by $3.4 \mathrm{~m}$ and are oriented back-to-back with no line-of-sight coupling between their apertures. The pulse response is measured using a real-time spectrum analyzer (Tektronix RSA 6114A) with acquisition bandwidth set to $40 \mathrm{MHz}$ and sampling time step $\delta t=20 \mathrm{~ns}$. The received train of the distorted pulse responses after being subjected to mode-stirred reverberation consists of 15000 quasi-periods (frames), each containing 5000 sampled I/Q pairs (Fig. 3). To analyze systematic phase effects, the pulse response in the static chamber ( $0 \mathrm{rps}$ ) was also measured across 15,000 quasi-periods, for reference. $^{2}$

Upward crossing times for the amplitude ${ }^{3}$ of the received pulses at an amplitude threshold level of $5 \sigma_{N}$ were extracted from the sampled data, where $\sigma_{N}$ is the standard deviation of the background noise amplitude during the preceding $60 \%$ offduty interval. The crossing times exhibit pulse-to-pulse variations ${ }^{4}$ that were found to contain the following components (Fig. 4):

1) quasi-periodic oscillation: caused by $50 \mathrm{~Hz}$ mains jitter with nominal average period $\left\langle T_{\tau}\right\rangle=200 T_{0}=20$

\footnotetext{
${ }^{2}$ Video clips of framed responses of the received pulse train during mode stirring at selected stir speeds are shown in the supplementary Multimedia files.

${ }^{3}$ Delays for threshold crossings of the I- and Q- components were found to produce characteristics that are noisier but otherwise similar to those for $|E|$, viz., $r\left(\mathcal{T}_{\operatorname{Re}(E)}, \mathcal{T}_{|E|}\right) \simeq r\left(\mathcal{T}_{\operatorname{Im}(E)}, \mathcal{T}_{|E|}\right)=0.98$ and $r\left(\mathcal{T}_{\operatorname{Re}(E)}, \mathcal{T}_{\operatorname{Im}(E)}\right)=0.95$ at $\Omega=0.25$ rps for the sample correlation coefficient $r\left(\mathcal{T}_{X}, \mathcal{T}_{Y}\right)$ between pulse delays $\mathcal{T}$ for $X$ and for $Y$.

${ }^{4} \mathrm{cf}$. video clips in the supplementary Multimedia files
}
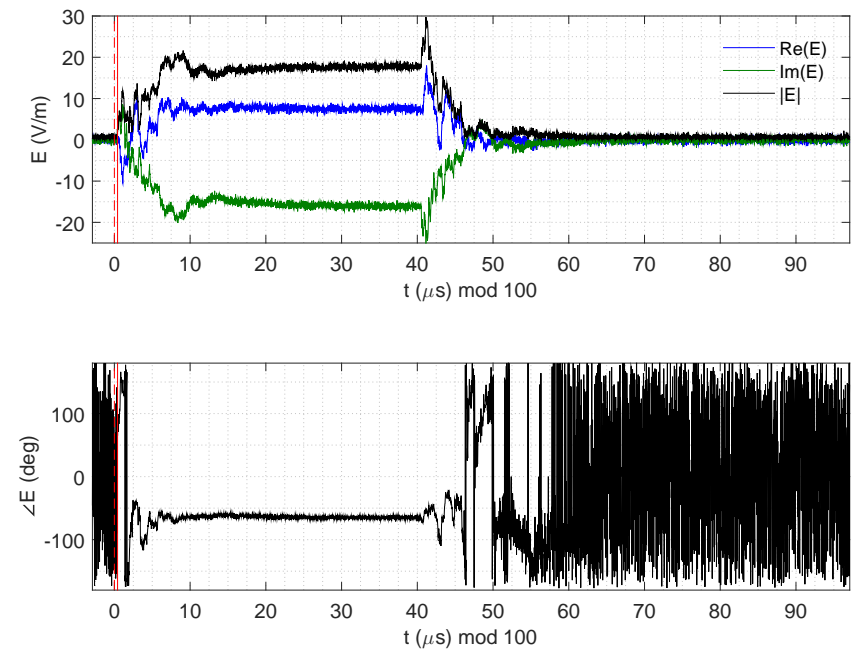

Fig. 3. Measured $\operatorname{Re}[E(t)], \operatorname{Im}[E(t)]$, amplitude $|E(t)|$ (top figure) and phase angle $\arg [E(t)]$ (bottom figure) for tenth frame $(i=10)$ in train of 15000 received pulse responses $(i=1, \ldots, 15000)$ at stir speed $\Omega=0.25 \mathrm{rps}$. Vertical marker (red solid line) at $t=21 \delta t=$ $0.42 \mu \mathrm{s}$ indicates delay $\tau_{10}$ of upward threshold crossing for $|E(t)|$ at level $5 \sigma_{N}$ for this frame, with reference to delay $\tau_{1} \triangleq 0$ in first frame (vertical red dashed line). See video clips in supplementary Multimedia files for sequence of pulse responses.

$\mathrm{ms}$, producing relatively slow fluctuations ${ }^{5}$ of the delays [25];

2) quasi-linear drift: independent of stir velocity, but increasing at a rate of approximately $10^{-6}(\sim 0.1 \mathrm{~ns}$ per pulse period); appearing in both dynamic and static cavity operation and attributable to drift by the pulse generator and in mains frequency;

3) small linear drift: attributable to stir speed and stir sense, causing Doppler phase shift (see below);

4) random component (jitter): caused by time-varying multipath propagation and any residual fluctuations.

Because of the known strong effect of filtering on jitter [27], no physical pre-filtering was applied at the transmitter side. Instead, drift and periodicity were separated from random timing fluctuations during post-processing of the pulse response data.

Component 2) yields the average rate of delay as the (scaled) average slope across all edge ${ }^{6}$ delays $\tau_{i}$ in Fig. 4a, viz., $\langle\mathrm{d} \tau / \mathrm{d} t\rangle=\left[\left\langle\tau_{i} / \delta t\right\rangle /\left(t / T_{0}\right)\right] / 5000$, where $T_{0}=5000 \delta t$. Fig. 5 shows that this rate, although small, oscillates with an increasing amplitude for increasing stir speed, and that its sign gets inverted consistently when reversing the sense of stirrer rotation, across a range of different measured stir speeds. These results are qualitatively consistent with the model in Sec. II (cf. Fig. 2); quantitative discrepancies can be ascribed to the $\Omega t$-dependence of the coefficients of $2 s \Omega / \lambda$

\footnotetext{
${ }^{5}$ After folding of the 75 edge quasi-periods, the relative correction for dilation is $2 \times 10^{-7}$, corresponding to just one sampling step time $\delta t$ per set of five edge quasi-periods.

${ }^{6}$ These do not necessarily coincide with the phase delays for a single harmonic plane wave or mode, hence these are now denoted as $\tau$ instead of $T$ in Sec. II
} 


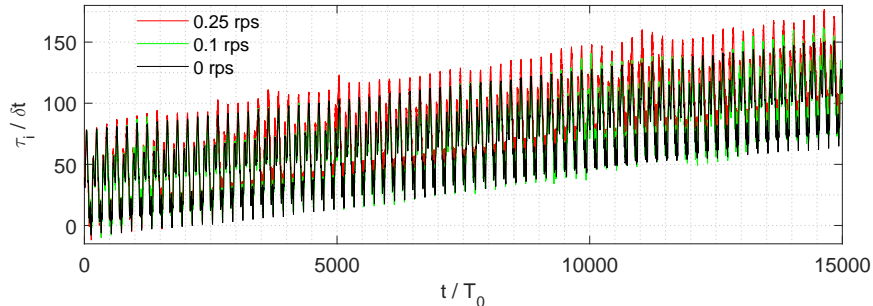

(a)

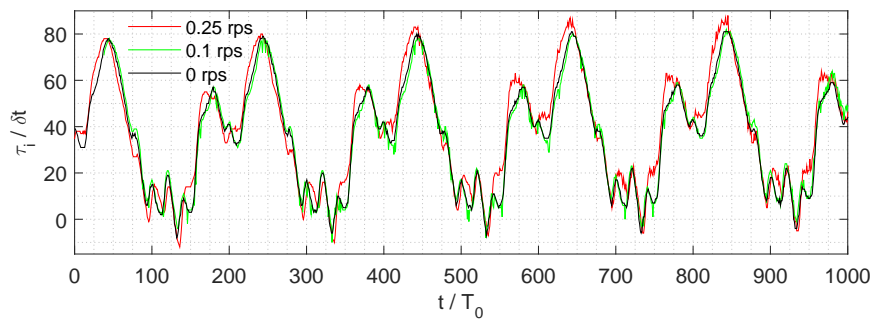

(b)

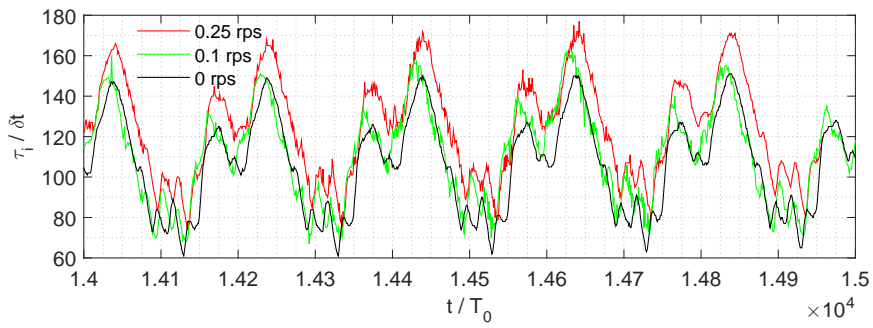

(c)

Fig. 4. (a) Time delays $\tau_{i}$ (in units $\delta t$ ) of all 15000 frames of received pulse responses for speeds zero (black), $0.1 \mathrm{rps}$ (green) and 0.25 rps (red), based on 5000 measured time samples per frame $(i=$ $1, \ldots, 15000)$. (b), (c) Close-up views: (b) first 1000 frames $\left(\mathcal{T}_{1}\right.$ to $\mathcal{T}_{5}$ ) and (c) last 1000 frames $\left(\mathcal{T}_{71}\right.$ to $\left.\mathcal{T}_{75}\right)$.

in (20), (22) and (24), as well as the uncertainty of $t$ and the fact that $\tau$ refers to threshold crossings of the pulse envelope, as opposed to phase delay in the sinusoidal carrier wave.

The threshold crossings exhibit a quasi-periodicity with

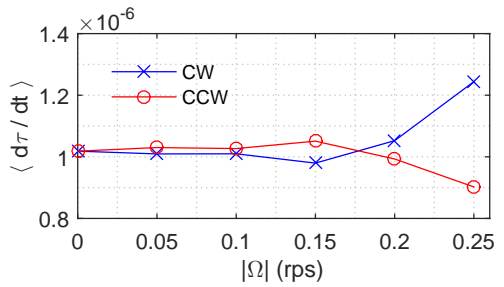

Fig. 5. Average rate of delay $\langle\dot{\tau}\rangle$ (scaled average slope from Fig. 4a) based on delay measurements for 11 applied stir velocities $(0 \ldots(0.05) \ldots 0.25 \mathrm{rps}$, each for clockwise $(\mathrm{CW})$ and for counterclockwise (CCW) rotation).

average period $\left\langle T_{\tau}\right\rangle$. Folding the 75 edge quasi-periods yields the minimum, average and maximum delays at ages $t \bmod \left\langle T_{\tau}\right\rangle$. Figs. 6a,b show these statistics for the excess delay $\tau-\tau_{\text {min }}$, where $\tau_{\text {min }} \triangleq \min _{i}\left(\tau_{i}\right)$ is the minimum minimorum delay. These delays show increased positive skewness for increased stir speed (average value lying closer to minimum than to maximum, for arbitrary $t$ ), indicating that stir parameter settings can be inferred from the statistics of the pulse delays. Increased speed also produces typically a smaller range $\max \left[\tau\left(t \bmod \left\langle T_{\tau}\right\rangle\right)\right]-\min \left[\tau\left(t \bmod \left\langle T_{r}\right\rangle\right)\right]$, mainly because of increased and smoother minimum excess delays. This suggests a stabilizing effect of stir speed on the spread of the phase noise per age within the pulse rise response. This is confirmed by Fig. 6c, showing progressively reduced standard deviations for increasing speeds. Beneficial effects of (limited) mode stirring on the performance of WLAN systems in a MSRC at some stir speeds have also been observed in [28, Fig. 5].

Subtracting the averages from the data at each age $i(\delta t)+(k-1)\left\langle T_{\tau}\right\rangle$ for $k=1, \ldots, 75$ and refolding yields the residual random fluctuations shown in Fig. 6d. These residuals are then analyzed statistically; a statistical model is developed hereafter. The residuals will further be denoted simply as $\tau_{i}$, i.e., centering (prior subtraction of $\langle\tau\rangle_{i}$ ) is tacitly assumed. The asymmetry in the spread of fluctuations for nonzero speed is also apparent from the data in Fig. 6d. Incidentally, such asymmetry in the spread of the pulse jitter has also been observed and modelled in a different EMC scenario and context [29, Figs. 8 and 11].

\section{Stochastic Model}

Mathematically, pulse jitter in mode-stirred reverberation may be viewed as a problem in optimal stopping theory, in which the first exit time of $|E(t)|$ can be estimated based on the formulaton and optimization of a so-called superharmonic reward function [30], [31]. Here, the reward is time-inhomogeneous because of the advent of pulse arrivals in a dynamic setting, which requires idealizations and approximations. Instead, we shall develop a heuristic approach, formulated in terms of arrivals of wave fronts and with a predefined time-independent threshold $\left(5 \sigma_{N}\right)$ for pulse detection.

Consider the complex-valued $E(t)$ during the edge of a pulse amplitude modulated carrier rising at $t=0$. In a quasi-static scenario, $E(t)$ can be considered as the resultant of the superposition of $N(t)$ multipath components ('rays'), representable by a random walk in the I/Q plane at the carrier frequency. In a simple statistical model for this multipath scenario, the capture of $n$ out of $N(t)$ rays by the receiving antenna with a matched direction of polarization constitutes $n$ successes in a set of $N(t)$ Bernoulli trials of waves impinging onto the antenna's effective aperture, each with a probability of success $p(t)$. For a fully developed pulse response $(t / \delta t \gg 1)$, the cavity field is stationary (cf. Fig. 3), hence $N$ and $p$ are then independent of time, leading to a homogeneous binomial process. When $N \gg 1$ whilst $p N \ll 1$ because of a relatively small aperture of the antenna compared to the cavity size, a homogeneous Poisson (HP) process for the captured rays results with a constant rate of $\operatorname{arrival}^{7} \lambda$.

\footnotetext{
${ }^{7}$ In Secs. IV and V, $\lambda$ now no longer symbolizes wavelength, as it does in Sec. II.
} 
The fields of the $n$ captured rays with interarrival times $\tau_{i}$ superimpose because of their nonzero life time as a result of the finite cavity relaxation time. Define the pulse arrival time as the aggregate $\mathcal{T}=\sum_{i=1}^{n} \tau_{i}=n / \lambda$ for the resultant amplitude of the fields to cross an arbitrary but sufficiently low amplitude threshold level, say $5 \sigma_{N}$. For independent and identically distributed (i.i.d.) ray arrivals in a HP model, $\mathcal{T}$ exhibits an Erlang gamma distribution

$$
f_{\mathcal{T}}(\tau)=\frac{\lambda^{n}}{\Gamma(n)} \tau^{n-1} \exp (-\lambda \tau)
$$

in which the rate $\lambda$ depends on the chosen amplitude threshold.

Fig. 7 shows histograms of the excess delay residuals $\tau_{i}-\min _{i}\left(\tau_{i}\right)$ in units $\delta t$ for the 15000 pulse edge responses, measured at $\Omega=0.1 \mathrm{rps}$ and $0.25 \mathrm{rps}$. These are compared with a best-fitting Erlangian $f_{\mathcal{T}}(\tau)$ for the HP model whose parameter values are estimated from the data as $n=$ $\left(m_{\mathcal{T}} / s_{\mathcal{T}}\right)^{2}=3.20$ and $2.78 ; \lambda=m_{\mathcal{T}} / s_{\mathcal{T}}^{2}=1.56 / \delta t$ and $1.62 / \delta t$, respectively, where $m_{\mathcal{T}}$ and $s_{\mathcal{T}}$ are the sample mean and standard deviation of $\mathcal{T}$. It is seen that an increased stir speed yields a narrower spread of the excess delay. Physically, this can be explained by the increased boundary dynamics assisting rays in impinging more successfully onto the receiving horn's effective aperture in a process of changing ray directions through stirring action, at an increasingly uniform (i.e., less time-dependent) rate as the received pulse approaches a steady state.

Unfortunately, since the steady-state mean free path travel time between consecutive reflections by the boundary $(4 V /(\mathrm{c} S) \sim 10 \mathrm{~ns})$ is smaller than $\delta t$, the earliest ray arrivals could not be detected and separated individually with the available instrumentation. Particularly for slower rotations that yield a larger delay spread and a smaller positive skewness (cf. Fig. 6), this limited measurement resolution causes overcrowding of the first and smallest possible data bin, i.e., for the earliest arrivals during the interval $[0, \delta t]$, as can be observed in Fig. 7a. The limited sampling resolution mainly affects the accuracy of the parameter estimation in the model curve fitting, typically by slightly overestimating $\lambda$ and underestimating the mode of the distribution at $\left(\tau_{i}-\tau_{\min }\right) / \delta t=(n-1) / \lambda$. In principle, this shortcoming can be mitigated through denser sampling (i.e., smaller $\delta t$ ), faster stirring (yielding smaller delay spread $\sqrt{n} / \lambda$ ), or using a larger cavity (larger $4 \mathrm{~V} / S$ ). Lowering the amplitude threshold level below $5 \sigma_{N}$ to reduce the count for the first data bin was found to cause a sharp rise of the false-alarm rate for the threshold crossing, making the manual correction for the extraction of statistics impractical.

In actuality, $\lambda$ must increase with time during the pulse rise, even in the absence of stirring [11], as this rate evolves from zero (no ray arrivals for $t<0$ ) to a steady-state value $\lambda_{\infty}$ for a fully developed pulse response. This can be explained by the fact that, during the reverberation time, noncaptured rays live on to become available again for further incidence onto the receiving antenna after missing their target and reflecting off static or dynamic boundary segments. This increases the interior ray density to produce an avalanche of rays. As a consequence, in steady state, $\lambda_{\infty}$ increases with $\xi_{S}$ and $\xi_{V}$.

A nonhomogeneous Poisson (NHP) process with a linear or linearized increasing rate $\lambda(t)=\lambda_{0}+\lambda_{1} t$ with $\lambda_{1}>0$ constitutes the simplest extension of a HP to a renewal process [32] with a more general $\lambda(t)$ but still governed by i.i.d. interarrivals. The parameters $\lambda_{0}$ and $\lambda_{1}$ measure the average speed and acceleration of path arrivals, respectively. The cases $\left(\lambda_{0} \neq 0, \lambda_{1}=0\right)$ and $\left(\lambda_{0}=0, \lambda_{1} \neq 0\right)$ correspond to constant rate and constant acceleration of arrivals, respectively. In an NHP process, the individual interarrival times $\mathcal{T}_{j}$ of the rays have an identical probability density function (PDF), viz.,

$$
\begin{gathered}
f_{\mathcal{T}_{j}}\left(\tau_{j}\right)=\frac{\exp \left(-\lambda_{0} \tau_{j}-\lambda_{1} \tau_{j}^{2}\right)}{\sqrt{\frac{\pi}{4 \lambda_{1}}} \exp \left(\frac{\lambda_{0}^{2}}{4 \lambda_{1}}\right)\left[1-\Phi\left(\frac{\lambda_{0}}{\sqrt{4 \lambda_{1}}}\right)\right]}, \\
0 \leq \tau_{j}<+\infty .
\end{gathered}
$$

The PDF $f_{\mathcal{T}}(\tau)$ then follows as the $n$-fold self-convolution of $f_{\mathcal{T}_{j}}\left(\tau_{j}\right)$, or equivalently using their Fourier transformations on account of the i.i.d. property, as

$$
\begin{aligned}
f_{\mathcal{T}}(\tau ; n)= & (2 \pi)^{-\frac{n+1}{2}}\left[1-\Phi\left(\frac{\lambda_{0}}{2 \sqrt{\lambda_{1}}}\right)\right]^{-n} \\
& \times \int_{-\infty}^{+\infty}\left[1-\Phi\left(-\frac{\mathrm{j} \omega}{2 \sqrt{\lambda_{1}}}\right)\right]^{n} \\
& \times \exp \left[-\frac{n \omega^{2}}{4 \lambda_{1}}+\mathrm{j} \omega\left(\frac{n \lambda_{0}}{2 \lambda_{1}}-\tau\right)\right] \mathrm{d} \omega(27)
\end{aligned}
$$

for $0 \leq \tau<+\infty$, with $\Phi(u) \triangleq(2 / \sqrt{\pi}) \int_{0}^{u} \exp \left(-x^{2}\right) \mathrm{d} x$ representing the error function. For $\tau \rightarrow+\infty$, this $f_{\mathcal{T}}(\tau)$ decreases according to $\exp \left(-\lambda_{0} \tau-\lambda_{1} \tau^{2} / n\right)$, to leading order in $\tau$. Thus, as the rate and uniformity of arrivals of $n$ impacting rays increases, the distribution of the delay times changes from a short-tailed $\chi_{1}$, i.e., semi-Gaussian PDF $\exp \left(-\tau^{2}\right)$ for $t \geq 0$ (i.e., $\left.n=0 ; \lambda_{0}=0, \lambda_{1} \neq 0\right)$ to the Erlangian $\exp (-\tau)$ for $t / \delta t \rightarrow+\infty$ (i.e., $n=n_{\infty} ; \lambda_{0} \neq 0, \lambda_{1}=0$ ). In an extended model, the fluctuation of $N$ with delay time can be accommodated by considering (27) to be a conditional PDF on $N$, which is then to be multiplied by a negative binomial probability mass function $f_{N}$ on $n$ in order to obtain $f_{\mathcal{T}}(\tau)$, but requiring a separate measurement or other independent evaluation of $N(t)$.

Fig. 8 compares the experimental complementary cumulative distribution function $(\mathrm{CCDF}) 1-F_{\mathcal{T}}(\tau)$ of $\mathcal{T}$ for 0.1 and $0.25 \mathrm{rps}$ against corresponding theoretical CCDFs for HP, NHP and semi-Gaussian processes. The figure confirms that a variable-rate NHP model (26) yields a considerably more accurate fit than the constant-rate HP process, particularly at lower stir speeds and for larger delays $\tau$. Since $\mathrm{d}\left[1-F_{\mathcal{T}}(\tau \rightarrow+\infty)\right] / \mathrm{d} \tau=-f_{\mathcal{T}}(\tau \rightarrow+\infty)$, first estimates of the parameters can be extracted by fitting the tail of the CCDF, followed by further nonlinear optimization to yield (27) using the simplex method.

For the lower stir speed $(0.1 \mathrm{rps})$, the ratio $\lambda_{1} /\left(n \lambda_{0}\right) \simeq 1.05$ is relatively large, indicating a significant increase in $\lambda(t)$, i.e., an accelerated growth of the number of captured rays during $[0, \mathcal{T}]$. The associated quasi-Gaussian CCDF behaviour for this lower speed is consistent with earlier measurement results for the RMS delay delay distribution of a static indoor propagation channel [33]. By contrast, for the higher speed ( $0.25 \mathrm{rps})$, the large- $\tau$ behaviour is now closer to the Erlang 
distribution for ideal HP arrivals, despite the similar value of $n$ as before. Here, the smaller value of $\lambda_{1} /\left(n \lambda_{0}\right) \simeq 0.29$ signifies a higher but more slowly increasing rate of arrival of the multipath components. This results in more effective field randomization per unit time, in a process whereby mode stirring (dynamic wave chaos) enhances the field mixing originating from unstirred reverberation (static wave chaos). This is confirmed by the direct comparison of the parameter values, based on more extensive data analysis for various speeds $(0.05 \leq \Omega \leq 0.25 \mathrm{rps})$ : lower stir speeds were found to exhibit generally smaller $\lambda_{0}$ and larger $\lambda_{1}$.

In summary, the NHP model with a linear $\lambda(t)$ and i.i.d. interarrival times offers a physical basis for graduation between the asymptotic short-tailed semi-Gaussian DF and Erlang DF for $\mathcal{T}$ when $\Omega$ and, consequently, the uniformity of the rate of ray arrivals are increased, resulting in improved mixing of the field as the pulse develops.

The present findings are also consistent with theoretical results [34] for a plane wave incident at an angle $\theta_{i}$ and reflected off a perfectly conducting plane that is oscillating mechanically at a rate $\Omega$. After expanding the incident pulse into an angular spectrum of plane-wave fields [35], each planewave component develops into a bundle of propagating or evanescent reflected plane waves that are spread across a set of discrete spatial reflection angles $\theta_{m}$ (similar to a frequency selective surface, e.g., [36]) at Fourier frequencies $\omega_{m}$ (as for normal incidence), governed by [34, eq. (38)]

$$
\theta_{m}=\sin ^{-1}\left(\frac{\omega_{0} \sin \theta_{i}}{\omega_{m}}\right), \quad \omega_{m}=\omega_{0}+m \Omega
$$

for the $m$ th spectral component, provided $k+m \Omega / \mathrm{c}>$ $\left(k \sin \theta_{i}\right)^{2}$. Although the interior of an overmoded MSRC is of course geometrically more complex than this single boundary in unbounded space, the qualitative picture is the same: the cavity boundary is locally plane in the high-frequency limit and each Fourier component $\Omega_{n}$ of the boundary motion, whether periodic or otherwise, produces side-band frequency components that lead to greater wave diffusion in space and in time. For a stirrer rotating at a constant speed, the contribution to the diffusion due to stir speed increases when $\Omega_{n}$ approaches the modal spectral spacing (reciprocal of the mode density).

Extending the previous analysis to a range of speeds shows that the estimated values of $n$ based on (27) are typically of the order $n \sim 1$ to 3 for the chosen threshold level. For $n=1$, (26) applies with $\mathcal{T}_{j} \equiv \mathcal{T}$; for $n=2$ and 3 , the double and triple self-convolutions of $f_{\mathcal{T}_{j}}\left(\tau_{j}\right)$ yield, with the aid of [37, eq. (3.322)],

$$
\begin{aligned}
& f_{\mathcal{T}}(\tau ; n=2)=\exp \left(-\lambda_{0} \tau-\frac{\lambda_{1}}{2} \tau^{2}\right) \Phi\left(\sqrt{\frac{\lambda_{1}}{2}} \tau\right) \\
& f_{\mathcal{T}}(\tau ; n=3)=\exp \left(-\lambda_{0} \tau-\frac{\lambda_{1}}{3} \tau^{2}\right) \\
& \times\left[\Phi\left(\sqrt{\frac{\lambda_{1}}{2}} \tau\right) \Phi\left(\sqrt{\frac{\lambda_{1}}{6}} \tau\right)\right. \\
& \left.-\sqrt{\frac{2 \lambda_{1}}{\pi}} \int_{0}^{\tau} \Phi\left(\sqrt{\frac{3 \lambda_{1}}{2}} x-\sqrt{\frac{2 \lambda_{1}}{3}} \tau\right) \exp \left(-\frac{\lambda_{1}}{2} x^{2}\right) \mathrm{d} x\right],
\end{aligned}
$$

up to respective normalization constants. Estimates of $n$ show a weak but, on average, progressive decrease with increasing stir speed, which is indicative of field "slipping" as the degree of nonstationarity increases [14].

Further insight can be gained by focusing on asymptotically large values of $\tau$ : in this case, the PDFs for $n=1,2$ or 3 can be combined into a single expression as

$$
\begin{aligned}
& f_{\mathcal{T}}(\tau\rightarrow+\infty ; n \leq 3) \propto \exp \left(-\lambda_{0} \tau-\frac{\lambda_{1}}{n} \tau^{2}\right) \\
& \quad \times\left[\Phi\left(\sqrt{\frac{(n-1) \lambda_{1}}{n}} \tau\right)+\Phi\left(\sqrt{\frac{\lambda_{1}}{n(n-1)}} \tau\right)\right](31)
\end{aligned}
$$

If we approximate the bracketed sum in (31) by a constant, on account of $\lim _{z \rightarrow+\infty} \Phi(z)=1-\exp \left(-z^{2}\right) /(\sqrt{\pi} z)$, then the $n$-fold convolution for any $n$ and $\tau \rightarrow+\infty$ follows as

$f_{\mathcal{T}}(\tau \rightarrow+\infty ; n)=\frac{\exp \left(-\lambda_{0} \tau-\frac{\lambda_{1}}{n} \tau^{2}\right)}{\sqrt{\frac{\pi n}{4 \lambda_{1}}} \exp \left(\frac{\lambda_{0}^{2} n}{4 \lambda_{1}}\right)\left[1-\Phi\left(\lambda_{0} \sqrt{\frac{n}{4 \lambda_{1}}}\right)\right]}$,

which requires renormalization when accounting for smaller values of $\tau$. For arbitrary $\lambda_{0}, \lambda_{1}>0$, this form shows the graduation from an NHP quasi-Gaussian to asymptotic HP tail behaviour when $n$ increases.

While the NHP process already offers an improved fit for the data, more sophisticated models can be envisaged. For example, the empirical CCDF for $0.25 \operatorname{rps}$ near $\left(\tau-\tau_{\min }\right) / s \mathcal{T} \simeq 5.5$ in Fig. 8b indicates that $f_{\mathcal{T}}(\tau)$ changes from quasi-Erlangian to semi-Gaussian in a rather abrupt manner. This suggests that excessively long delay "outliers" may be associated with rays in a NHP process that have not (yet) been sufficiently uniformly mixed (understirred or static path fields). Such twotier behaviour of the CCDF that depends on the magnitude of the delay suggests an extension of the NHP model, either (i) locally, to yield a composite process whereby different rate parameters $\lambda_{0,1}^{(i)}$ apply during different subintervals $\left[\tau_{i}, \tau_{i+1}\right]$, in the same vein as in hypoexponential distributions [38], or (ii) globally, to produce a higher-order polynomial expansion $\lambda(t)=\sum_{\ell=0}^{L} \lambda_{\ell} t^{\ell}$ with time-varying rates of velocity and acceleration for pulse arrivals), with which a shortening of the right tail of $f_{\mathcal{T}}(\tau)$ and hence $1-F_{\mathcal{T}}(\tau \rightarrow \infty)$ can be achieved through a nonuniform weighting of the different sections of the CCDF by the $\lambda_{\ell}$.

\section{CONCLUSIONS}

A time-domain technique was developed for evaluating the randomization (mixing) of a continuously mode-stirred cavity field generated by a pulse modulated source. The results are particularly important for the estimation of phase errors and more general timing instabilities, including their fluctuations and uncertainties. These are of immediate relevance in wireless digitally modulated signals propagating in dynamic multipath EMEs, with a view to improve synchronization, to reduce intersymbol and co-channel interference and symbol error rates (e.g., in COFDM), and to shorten guard intervals for the benefit of increasing channel capacity or quality of service. Furthermore, since phase statistics govern the degree of coherence and the instantaneous energy, the method can 
also lead to a more accurate characterization of transient random fields, in their evolution from background noise to fully developed wave chaos, which finds application in the stochastic characterization of the early power delay profile and outage. As such, the results are also relevant to wireless power transfer at radio frequencies.

Because of the arbitrary level of the detection threshold, the method entails an amplitude-free evaluation of the phase noise for the rising edge of the received pulse response. Systematic effects (phase drift) are traceable to deterministic parameters of the stir process (constant speed and sense of rotation of the mode stirrer), whereas the random phase noise yields the rate of the field randomization per unit time (stir quality). Both quantitative and qualitative differences in the PDF of the pulse delay are governed by the stir velocity.

Supported by a nonhomogeneous Poisson model, a transition was observed from a low but rapidly increasing rate of ray arrivals $\lambda(t) \simeq \lambda_{1} t$ at low stir speeds toward higher but more stable rates $\lambda(t) \simeq \lambda_{\infty}$ at higher stir speeds, together with an additional variance stabilization of delays at arbitrary times during the pulse transient. These are manifestations of the increasing rate of field mixing per unit time. This velocityand hence time-dependence of the mixing rate suggests a more general functional form

$$
\lambda(t, \Omega)=\lambda_{\infty}[1-\exp (-\mu(\Omega) t)]
$$

for further refining the dynamic model. More generally, the higher-order rate dependence offers an avenue for physical modelling of short-tailed Weibull or Box-Tiao generalized exponential distributions, also in other engineering applications. The accuracy of the NHP parameter estimation from measured data is governed by the time resolution for measuring the minimum delays, associated with the first few reflections near the onset of the pulse. Increased sampling rates by future instrumentation should enable characterization of such higherorder models with increasing accuracy.

\section{ACKNOWLEDGEMENTS}

Measurements were supported by the 2006-2009 Electrical Programme of the U.K. National Measurement Systems Policy Unit. The spectrum analyzer was made available by courtesy of M. Vincent (Tektronix UK).

\section{REFERENCES}

[1] O. Bohigas, M. J. Giannoni, and C. Schmit, "Characterization of chaotic quantum spectra and universality of level fluctuation laws," Phys. Rev. Lett., vol. 52, no. 1, pp. 1-4, 1984.

[2] L. R. Arnaut, "Limit distributions for imperfect electromagnetic reverberation," IEEE Trans. Electromagn. Compat., vol. 45, no. 2, pp. 357377, May 2003.

[3] B. Dietz, A. Heine, A. Richter, O. Bohigas, and P. Lebœuf, "Spectral statistics in an open parametric billiard system" Phys. Rev. E, vol. 73, 035201(R), 2006.

[4] J.-B. Gros, U. Kuhl, O. Legrand, F. Mortessagne, O. Picon, and E. Richalot, "Statistics of the electromagnetic response of a chaotic reverberation chamber," Adv. Electromagn., vol. 4, no. 2, pp. 38-43, 2015.

[5] J. Koiller, R. Markarian, S. O. Kamphorst, and S. P. de Carvalho, "Time dependent billiards," Nonlinearity, vol. 8, no. 6, pp. 983-1003, Nov. 1995.
[6] D. Lubman, "Spatial averaging in a diffuse sound field," J. Acoust. Soc. Am., vol. 46, pp. 532-534, 1969.

[7] C. Ebbing, "Experimental evaluation of moving sound diffusers for reverberation rooms," J. Sound Vibr., vol. 16, pp. 99-118, 1971.

[8] J. Tichy and P. K. Baade, "Effect of rotating diffusers and sampling techniques on sound-pressure averaging in reverberation chambers," $J$. Acoust. Soc. Am., vol. 56, no., 1, pp 137-143, Jul. 1974.

[9] V. Rajamani, C. F. Bunting and J. C. West, "Stirred-mode operation of reverberation chambers for EMC testing," IEEE Trans. Instr. Meas., vol. 61, no. 10, pp. 2758-2764, Oct. 2012.

[10] J. Kim, J. Chen, J. Cox and F. X. Kärtner, "Attosecond-resolution timing jitter characterization of free-running mode-locked lasers", Opt. Lett., vol. 32, no. 24, pp. 3519-3521, Dec. 2007.

[11] L. R. Arnaut, D. A. Knight, "Observation of coherent precursors in pulsed mode-stirred reverberation fields," Phys. Rev. Lett., vol. 98, no. 5, 053903, Feb. 2007.

[12] L. R. Arnaut, "Statistical characterization of dynamic propagation environments for mobile wireless communications systems," Proc. XIXth IMEKO World Congr., Lisbon, Portugal, pp. 2315-2318, Sep. 2009.

[13] L. R. Arnaut and P. D. West, "Evaluation of the NPL untuned stadium reverberation chamber using mechanical and electronic stirring techniques," NPL Report, CEM 11, Nat. Phys. Lab., Teddington, U.K., ISSN 1369-6742, Aug. 1998.

[14] L. R. Arnaut, "Nonstationary random acoustic and electromagnetic fields as wave diffusion processes," J. Phys. A: Math. Gen., vol. 40, no. 27 pp. 7745-7788, Jul. 2007.

[15] G. Gradoni and L. R. Arnaut, " Transient evolution of eigenmodes in dynamic cavities and time-varying media," Radio Sci., vol. 50, RS005795, Oct. 2015.

[16] F. Mortessagne, O. Legrand and D. Sornette, "Role of the absorption distribution and generalization of the exponential reverberation law in chaotic rooms," J. Acoust. Soc. Am., vol. 91, no. 1, pp. 169-178, 1994.

[17] D.-H. Kwon, R. J. Burkholder and P. H. Pathak, "Ray analysis of electromagnetic field build-up and quality factor of electrically large shielded enclosures," IEEE Trans. Electromagn. Compat., vol. 40, no. 1, pp. 19-26, Feb. 1998.

[18] G. Castaldi, V. Galdi and I. M. Pinto, "Short-pulsed wavepacket propagation in ray-chaotic enclosures," IEEE Trans. Ant. Propag., vol. 60, no. 8, pp. 3827-3837, Aug. 2012.

[19] C. L. Holloway, H. A. Shah, R. J. Pirkl, K. A. Remley, D. A. Hill, and J. Ladbury, "Early time behavior in reverberation chambers and its effect on the relationships between coherence bandwidth, chamber decay time, RMS delay spread, and the chamber buildup time," IEEE Trans. Electromagn. Compat., vol. 43, no. 4, pp. 714-725, Aug. 2012.

[20] L. R. Arnaut, "Time-domain characterization of mode-stirred operation performance: can we do it?", presented at 2nd Nottingham-Nice Days Int. Workshop on Wave Complexity, Nottingham, U.K., 1-3 Jul. 2015.

[21] C. Møller, The Theory of Relativity. 2nd revised ed., Oxford University Press: London, U.K., 1976.

[22] P. T. Jensen, A. P. Mynster, and R. B. Behnke, "Practical industrial EUT testing in reverb chamber: experiences, findings and practical observations on high amplitude immunity testing of industrial equipment in reverberation chamber," Proc. EMC Europe 2014 Int. Symp. Electromagn. Compat., Gothenburg, Sweden, Sep. 2014, pp. 274-279.

[23] K. Karlsson, X. Chen, P.-S. Kildal, and J. Carlsson, "Doppler spread in reverberation chamber predicted from measurements during stepwise stationary stirring," IEEE Ant. Wireless Propag. Lett., vol. 9, pp. 497500, 2010.

[24] L. R. Arnaut and G. Gradoni, "Probability distribution of the coherence bandwidth of a mode-stirred reverberation chamber," IEEE Trans. Ant. Propag., vol. 63, no. 5, pp. 2286-2290, May 2015.

[25] http://www.nationalgrid.com/uk/Electricity/Data/Realtime/Frequency/ Freq60.htm.

[26] R. E. Richardson, "Mode-stirred chamber calibration factor, relaxation time and scaling laws," IEEE Trans. Instr. Meas., vol. 34, no. 4, pp. 573-580, 1985.

[27] L. E. Franks and J. P. Bubrouski, "Statistical properties of timing jitter in PAM timing recovery scheme," IEEE Trans. Circ. Syst., vol. 21, no. 4, pp. 489-496, Jul. 1974.

[28] R. Recanatini, F. Moglie, and V. Mariani Primiani, "Performance and immunity evaluation of complete WLAN systems in a large reverberation chamber," IEEE Trans. Electromagn. Compat., vol. 55, no. 5, pp. 806-815, Oct. 2013.

[29] M. P. Robinson, K. Fischer, I. D. Flintoft, and A. C. Marvin, "A simple model of EMI-induced timing jitter in digital circuits, its statistical distribution and its effect on circuit performance," IEEE Trans. Electromagn. Compat., vol. 45, no. 3, pp. 513-519, Aug. 2003. 
[30] E. B. Dynkin, "The optimum choice of the instant for stopping a Markov process," Sov. Math., vol. 4, pp. 627-629, 1963.

[31] B. Øksendal, Stochastic Differential Equations. 5th ed., Springer Verlag: NY, 2000.

[32] L. Kleinrock, Queuing Systems, vol. I. Wiley: NY, 1975.

[33] H. Hashemi and D. Tholl, "Statistical modelling and simulation of the RMS delay spread of indoor radio propagation channels," IEEE Trans. Veh. Techn., vol. 43, no. 1, pp. 110-120, Feb. 1994.

[34] D. De Zutter, "Reflection from linearly vibrating objects: plane mirror at oblique incidence," IEEE Trans. Ant. Propag., vol. 30, no. 5, pp. 898-903, Sep. 1982.

[35] L. R. Arnaut, "Angular spectral plane-wave decomposition of nonstationary random fields in mode-stirred reverberation," Phys. Rev. E, vol. 81, no. 4, 041133, Apr. 2010.

[36] J. F. Blackburn and L. R. Arnaut, "Numerical convergence in periodic method of moments analysis of frequency-selective surfaces based on wire elements, IEEE Trans. Ant. Propag., vol. 53, no. 10, pp. 3308-3315, Oct. 2005.

[37] I. S. Gradshteyn and I. M. Ryzhik, Table of Integrals, Series, and Products, 7th ed. Academic Press: NY, 2007.

[38] W. Feller, An Introduction to Probability Theory and its Applications, vol. II, ch. 1. Wiley:NY, 1971.

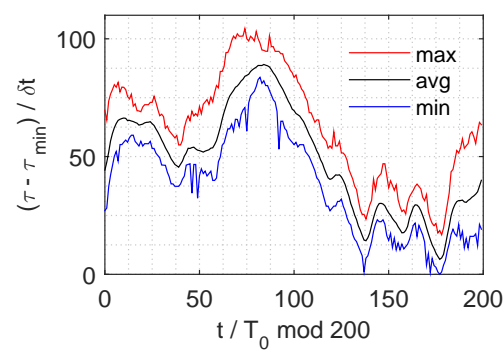

(a)

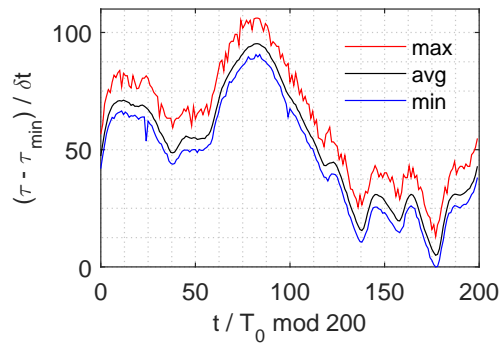

(b)

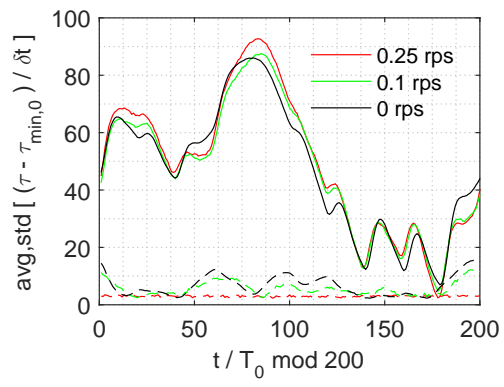

(c)

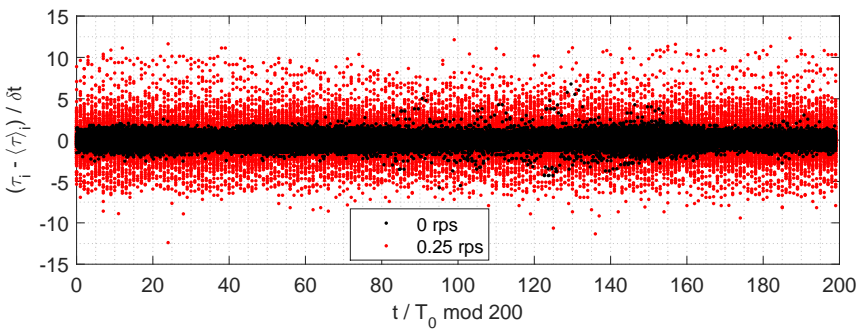

(d)

Fig. 6. (a), (b) Maximum $\max \left[\tau\left(t \bmod \left\langle T_{r}\right\rangle\right)\right]$, average $\langle\tau\rangle_{i}$, and minimum $\min \left[\tau\left(t \bmod \left\langle T_{r}\right\rangle\right)\right]$ excess delay times (in units $\delta t$ ) for (a) $\Omega=0.1 \mathrm{rps}$ and (b) $\Omega=0.25 \mathrm{rps}$, after folding 75 quasi-periods of 200 pulse edge starting times $\left(\left\langle\mathcal{T}_{\tau}\right\rangle=200(5000 \delta t)=20 \mathrm{~ms}\right)$, with reference to $\tau_{\min } \equiv \min _{i}\left[\tau_{i}(\Omega)\right]$. (c) Averages $\langle\tau\rangle_{i}$ (solid lines) and standard deviations $s_{\tau_{i}}$ (dashed lines) of delays in units $\delta t$ per age across the folded quasi-periods, for $\Omega=0$ (black), $0.1 \mathrm{rps}$ (green), and $0.25 \mathrm{rps}$ (red), with reference to $\tau_{\min , 0} \triangleq \min _{i}\left[\tau_{i}(\Omega=0)\right]$. (d) Residual delays (phase noise) in units $\delta t$ after subtracting averages $\langle\tau\rangle_{i}$ of folded responses from data and removal of periodicity and drift. 


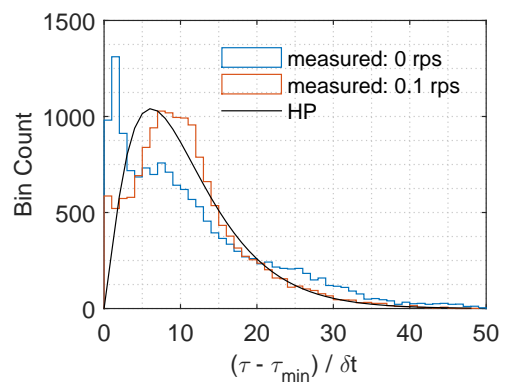

(a)

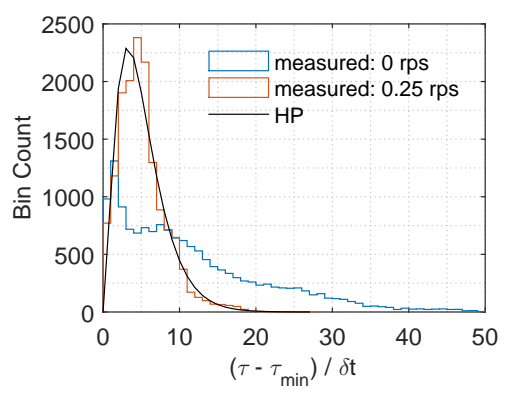

(b)

Fig. 7. Histogram of excess delay residuals $\tau_{i}-\tau_{\min }$ in units $\delta t$ (bin widths), for static cavity (blue) and (a) for $\Omega=0.1 \mathrm{rps}$ (red) $\left(m_{\mathcal{T}}=\right.$ $\left.10.88 \delta t, s_{\mathcal{T}}=7.19 \delta t\right)$; (b) for $\Omega=0.25 \mathrm{rps}$ (red) $\left(m_{\mathcal{T}}=5.14 \delta t\right.$, $s_{\mathcal{T}}=3.13 \delta t$ ). Logarithmically best-fitting scaled Erlang PDFs for homogeneous Poisson (HP) model (black) are shown for comparison.

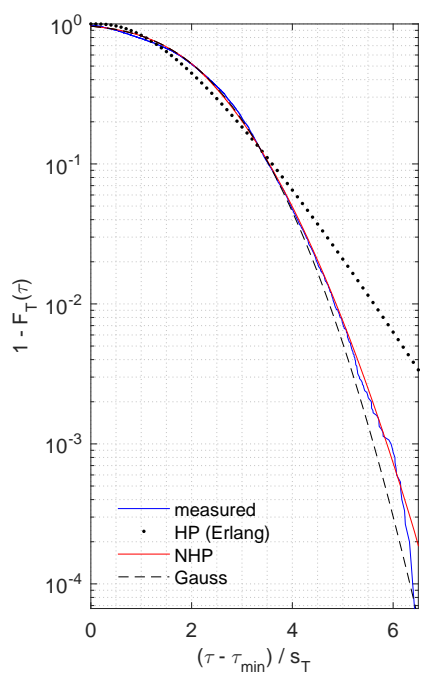

(a)

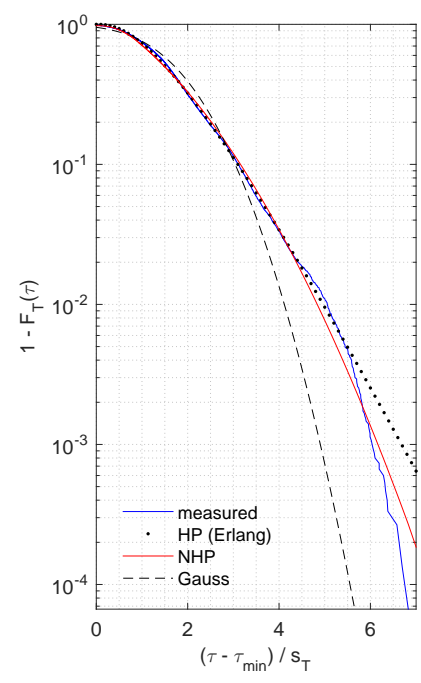

(b)
Fig. 8. (a) Empirical CCDFs (blue) of standardized excess delay residuals for $\Omega=0.1 \mathrm{rps}$ compared with CCDF of logarithmically best-fit nonhomogeneous Poisson process (red), for linear rate of interarrival $\lambda_{0}+\lambda_{1} t$ with $n=2.98, \lambda_{0}=0.22, \lambda_{1}=0.69$ in units $\delta t$ and $(\delta t)^{2}$. Dotted curve (black) represents CCDF for Erlang gamma homogeneous Poisson model $(n=3.20, \lambda=$ 1.56); dashed curve (black) shows asymptotic semi-Gaussian CCDF $1-\left[1+\Phi\left(\left(\tau^{*}-m_{\mathcal{T}^{*}}\right) /\left(\sqrt{2} s_{\mathcal{T}^{*}}\right)\right)\right] / 2$ valid for $\Omega \rightarrow 0$, based on empirical $m_{\mathcal{T}^{*}}=2.06$ and $s_{\mathcal{T}^{*}}=1.15$ at $0.1 \mathrm{rps}$, where $\mathcal{T}^{*} \triangleq\left(\mathcal{T}-\tau_{\min }\right) / s_{\mathcal{T}}$. (b) Same as figure (a) for $\Omega=0.25 \mathrm{rps}$, with logarithmically best-fit NHP model $\left(n=2.61, \lambda_{0}=0.41\right.$, $\left.\lambda_{1}=0.31\right)$, asymptotic HP model $(n=2.78, \lambda=1.62)$ valid for $\Omega \rightarrow \infty$, and semi-Gaussian model $\left(m_{\mathcal{T}^{*}}=1.72, s_{\mathcal{T}^{*}}=1.03\right.$ at $0.25 \mathrm{rps})$. 